\title{
Deflections and Strains in Cracked Shafts Due to Rotating Loads: A Numerical and Experimental Analysis
}

\author{
Nicolò Bachschmid and Ezio Tanzi \\ Dipartimento di Meccanica, Politecnico di Milano, Milan, Italy
}

In this article the deflections of a circular cross-section beam presenting a transverse crack of varying depths caused by various loads (bending, torsion, shear, and axial loads) are analyzed with the aid of a rather refined three-dimensional model that takes into account the nonlinear contact forces in the cracked area. The bending and shear loads are applied in several different angular positions in order to simulate a rotating load on a fixed beam or, by changing the reference system, a fixed load on a rotating beam. Torsion and axial loads are fixed with respect to the beam.

Results obtained for the rotating beam can then be used for the analysis of cracked horizontal-axis heavy rotors in which the torsion is combined with the bending load. The effect of friction is also considered in the cracked area. The characteristic "breathing" behavior of the cracked area was analyzed and compared to that obtained with a rather simple one-dimensional model. The differences in results with respect to those based on fracture mechanics are emphasized. In order to highlight the effect of the presence of the crack, the deflections of the uncracked beam loaded with the same loads were subtracted from the deflections of the cracked beam.

Finally, a cracked specimen was extensively analyzed by means of several strain gauges to study the strain distribution on the outer surface around the crack in various loading conditions. Consistent pre-stresses were found, and they influence the breathing behavior. The experimental

Received 28 March 2002; accepted 28 March 2002.

The financial support of MURST cofinanziamento "Identificazione di malfunzionamenti nei sistemi meccanici" (1999) is gratefully acknowledged.

Address correspondence to Nicolò Bachschmid, Dipartimento di Meccanica, Politecnico di Milano, Via la Masa, 34, I-20158 Milano, Italy. E-mail: nicolo.bachschmid@polimi.it results were compared with those obtained using the onedimensional linear model.

Keywords Cracks, Shafts

The behavior of a crack in a rotating shaft can be modeled by various methods, as reported in the literature. In this study, an original simplified linear model that allows cracks of various shapes to be modeled was used for calculating deflections of cracked beams in a variety of loading conditions. The results were compared with those obtained using two other methods: a cumbersome three-dimensional, (3D) nonlinear, finite element model, and a model obtained by using the release rate of the strain energy as determined by fracture mechanics. The comparison allowed evaluation of the accuracy of the methods with respect to the 3D model. Finally, experimental results obtained from a cracked specimen showed some unexpected effects, which can also be simulated easily by the simplified model.

\section{DESCRIPTION OF THE TEST BEAM}

The test beam used for the validation was a circular crosssection beam with diameter $d=25 \mathrm{~mm}$ and length $l=50 \mathrm{~mm}$, with a crack of varying shape in the middle. The beam is represented in Figure 1 together with the reference frame according to which the deflections were evaluated. The beam was clamped at one end, and a bending moment of $M_{b}=10 \mathrm{Nm}$ and a torsion of $M_{t}=25 \mathrm{Nm}$ were applied to the other end. Also a shear force $T$ of $1000 \mathrm{~N}$ was applied in some cases at the free end of the clamped beam to investigate its effects, and an axial force $N$ of $1500 \mathrm{~N}$ was applied in some other cases. Deflections were evaluated at the same end of the beam. In the 3D model, in order to avoid local deformations in the section where the deflections were evaluated, the beam had an extension, and the loads were applied to the end of this extension. The bending moment was then rotated in the direction of $\Omega$. 


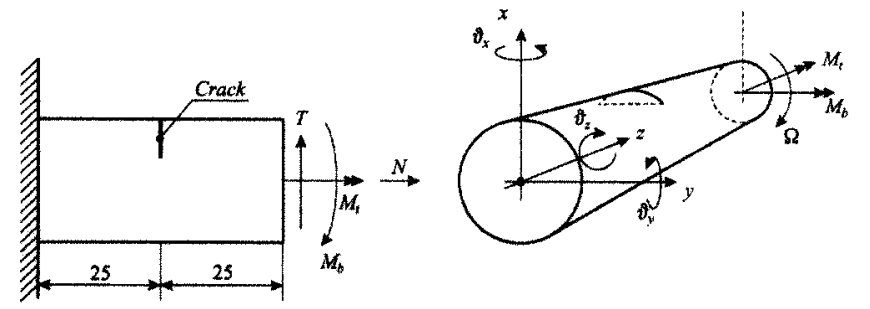

FIGURE 1

Test beam.

\section{DESCRIPTION OF THE MODEL}

The deflections of a test specimen were calculated by means of three different models: a 3D finite element model, a model based on fracture mechanics, and the simplified one-dimensional (1D) model developed by the authors.

\section{The 3D Model}

Figures 2 and 3 show the mesh that was used for the cracked cylinder, with relative crack depths of $25 \%$ and $50 \%$. Roughly 9000 and 11,000 elements, respectively, were used for the analysis of the two cracked cylindrical beams. A rather dense mesh was chosen because not only deformations of the cracked specimen, but also stress intensity factors in correspondence with the crack's tip were calculated numerically and compared with those calculated by means of classical fracture mechanics. This comparison allowed evaluation of the accuracy of the model in terms of its ability to represent real crack behavior in the region close to the crack. The elastic limit was never exceeded in the simulations.

The contact model in the cracked surface was obviously nonlinear. A friction coefficient $(f=0.2)$ was introduced in order to account for microslip conditions in the cracked area caused by shear forces and torsion. To avoid local deformations due to the application of loads, the model was extended, and a pure bending load was applied to the specimen. Thus, in the cracked area and in the measuring section, where the deflections were evaluated (indicated by the dashed line in Figs. 2 and 3), no local deformations were present because of the application of loads. The results obtained with this model are called simply the 3D results.

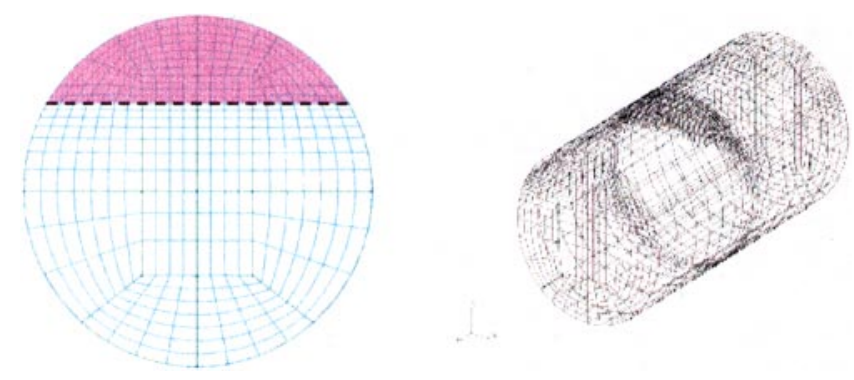

FIGURE 2

Mesh of the section and isometric view of the model with a crack of $25 \%$. The crack's tip is indicated by the dashed line.
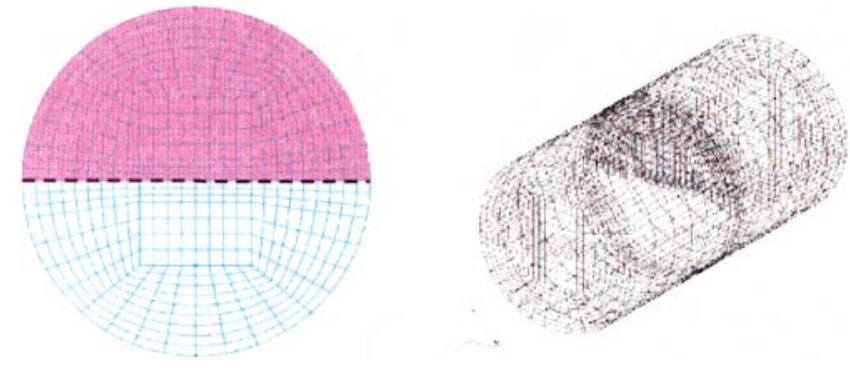

FIGURE 3

Mesh of the section and isometric view of the model with a crack of $50 \%$. The crack's tip is indicated by the dashed line.

\section{The Strain Energy Release Rate}

Because the strain energy release rate (SERR) approach combined with the stress intensity factors had been used by many authors (Dimarogonas 1996; Gasch 1998; Wauer 1990) for calculations of cracked beam bending behavior, several calculations were made using this approach and applying it to various crack depths. In this case, the breathing mechanism was assumed to be known (from the finite element model or from the simplified model), and the SERR approach was applied to the cracked cross-section, with its open and closed portions, in order to calculate the bending stiffness of the beam, as described in detail by Ferrari and Campana (1999). The extension of this approach to the breathing crack was affected by some errors: the crack's tip is supposed to be formed by the boundary between the cracked areas and the uncracked areas for the regions in which the breathing crack is open, which is correct; and by the boundary between the closed cracked areas and the open cracked areas, which is not correct, because on this boundary no stress intensity factors appeared.

The traditional approach is relatively simple and the nodal model allows the local character of the crack to be considered. The values of the terms of the stiffness matrix of the crack element depend on the open or closed configuration of the crack, which can be evaluated by comparing the sense of the displacement of the crack faces with the sense of the normal vector at the crack face. This approach is useful for evaluating the stress distribution in the cracked section; however, it does not consider the effects of thermal stress on the breathing mechanism of the crack, and the breathing itself is only roughly estimated, as the crack is considered completely open or closed. As far as the authors know, the breathing mechanism was simulated in previous studies by assigning a preestablished analytical law of the stiffness variation over the rotation. In this investigation, the nodal model combined with other crack models that take into account the breathing mechanism, were used to obtain results that would be compared with the cumbersome $3 \mathrm{D}$ calculations and with the results of the simplified model.

This approach assumes stress and strain distributions with same values in directions parallel to the applied bending moment axis (as they are in rectangular cross-sections) and no 


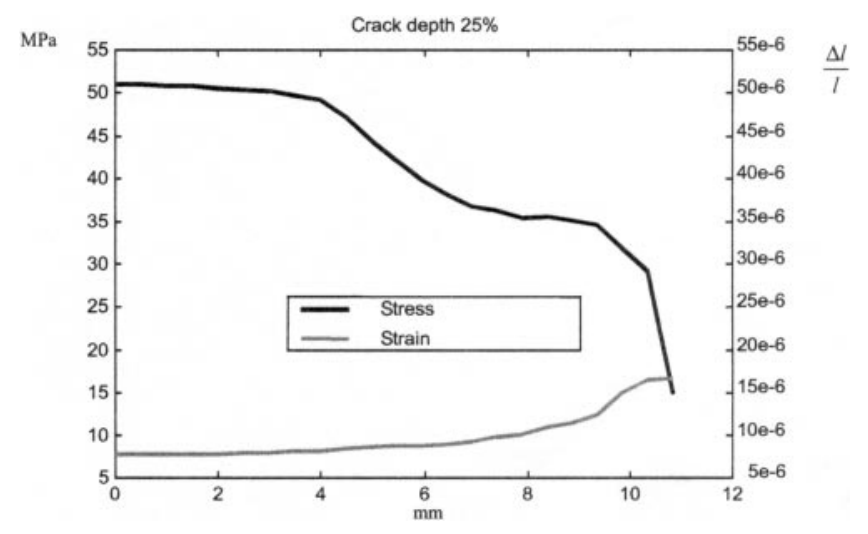

FIGURE 4

Distribution of strains and stresses along the tip of a $25 \%$ crack depth.

collaboration between the parallel rectangular slices into which the circular cross-section was divided. This is not realistic, as is shown in Figure 4, where the stress and strain along the crack tip are indicated as derived by using the $3 \mathrm{D}$ calculation. The cracked cross-section is no longer planar but is distorted. This is not taken into account by the fracture mechanics approach. What's more, the fracture mechanics approach does not consider any friction on the cracked area, and this also seems to be unrealistic. If torsion is present, the effects of friction forces on the cracked area can be taken into account only by the nonlinear 3D calculation and, in an approximate way, by the simplified model. The results obtained using this model are called the SERR results.

\section{The 1D Model}

The crack model (Bachschmid et al. 2000) is composed of a simplified equivalent beam model and a simplified model of the breathing mechanism, as a function of the static bending moment stresses. At the points of the cracked area where the stresses are compressive, contact occurs between the two faces of the crack; where the stresses are instead tensile, no contact occurs. By considering the sign of the stresses, the open and closed parts of the cracked area are determined in the various angular positions. The procedure is obviously roughly approximated because the actual stress distribution over the cracked cross-section due to the bending moment is not at all linear (as is assumed by the simplified model). The closed parts of the cracked area and the uncracked area contribute to the second moment of the area, which determines the stiffness of the equivalent beam; that, in turn, is a function of the angular position of the rotor with respect to the fixed weight load. This simple 1D model of the crack behavior is called FLEX model for brevity. The results using this model are called the (FLEX) results.

\section{THE BREATHING BEHAVIOR}

The breathing mechanism is generated by the bending moment caused by external loads such as the forces of weight and bearing reaction. The bending moment of $10 \mathrm{Nm}$ generated a maximum axial stress of $6.5 \mathrm{~N} / \mathrm{mm}^{2}$ on the outer cylinder surface. The breathing is produced by the stress distribution around the crack. Therefore, the question arises: Is the breathing also influenced by the shear stresses due to the external torsion moment, which certainly changes the stress distribution around the crack? To answer this question in the 3D model of the cracked beam, a rather high torsion moment was applied to emphasize the effect.

The torsion moment of $\mathrm{Mt}=25 \mathrm{Nm}$ generated a maximum tangential stress that was 1.25 times the maximum axial stress due to the bending moment of $10 \mathrm{Nm}$. In comparing the results obtained in the case of bending plus torsion loading to results obtained in the case of pure bending loading in the various angular positions, it was shown that the effect of torsion loads on the breathing mechanism of cracks can be neglected.

Even if the highly nonlinear stress distribution in the 3D finite element model is different from that in the linear (simplified) model, the two models actually showed rather good agreement in terms of the breathing mechanism for both the $25 \%$ - and the 50\%-depth cracks.

The breathing behavior allowed calculation of the areas and the second moments of areas of the cracked section; these were used for calculating the stiffness of the cracked beam in the various angular positions. Applying various loads, the deflections could then be calculated.

\section{DEFLECTIONS}

In order to emphasize the additional deflections caused by the presence of the crack alone, the deflections in the cracked specimen were compared to the deflections in the uncracked specimen, and its differences extracted. This procedure allowed for appreciation of small differences in behavior due to the various methods of modeling the crack.

The deflections were calculated in the center of the crosssection of the circular end of the specimen modeled with finite beam elements, according to the FLEX model and to the SERR model derived from fracture mechanics. All six components of the deflection were calculated for the various angular positions of the load with respect to the crack; they were obtained by increasing the angle in steps of 30 degrees.

Two different crack shapes were considered: a rectilinear crack with a relative depth of $25 \%$ and a rectilinear crack with a relative depth of $50 \%$.

Bending, shear, torsion, and axial loads were applied to the same specimen in order to simulate all possible loads that would occur in rotating shafts while in operation.

Finally, all results were compared.

\section{Pure Bending}

In Figures 5 and 6 it can be seen that the results of the FLEX model are rather close to the results of the 3D model-closer 


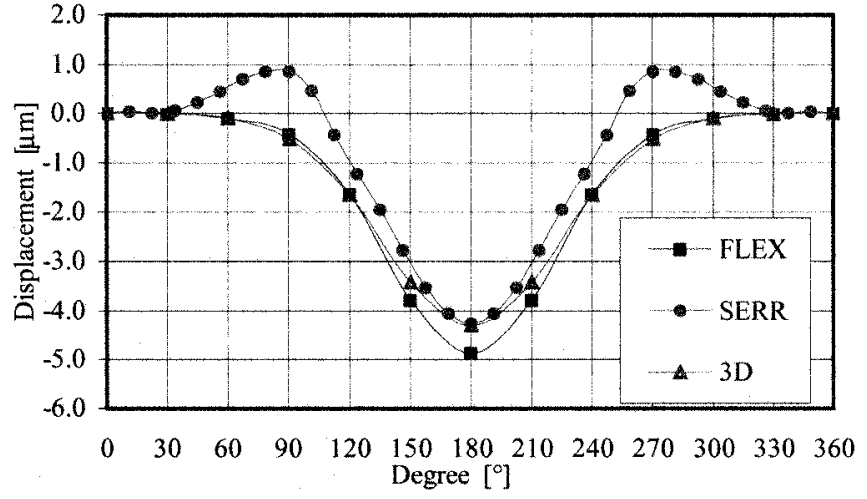

FIGURE 5

Pure bending, $50 \%$ crack depth, $\times$ displacement.

than are the SERR results. Similar behaviors were found for the other degrees of freedom, $y$ and $\vartheta_{y}$.

Only the axial displacement, $z$ (Fig. 7), due to the coupling effects is underestimated; the torsional deflection (Fig. 8) due to the coupling effects is overestimated by the FLEX model. But the differences (errors) are so small with respect to the other deflections that they can be neglected. Similar results were also found for the $25 \%$ crack.

\section{Bending and Torsion}

Because of the presence of torsion, the diagrams are no longer symmetrical. Again the FLEX results are closer to the 3D results than to the SERR results, as shown in Figures 9 and 10. In particular, the torsional flexibility is completely underestimated by SERR, as shown in Figure 11. The axial displacement (not shown) seems unaffected by torsional loads and is exactly equal to that of the pure bending case.

\section{Bending and Shear}

The presence of shear forces slightly lowers the accuracy of the FLEX model in general, except for the axial displacement,

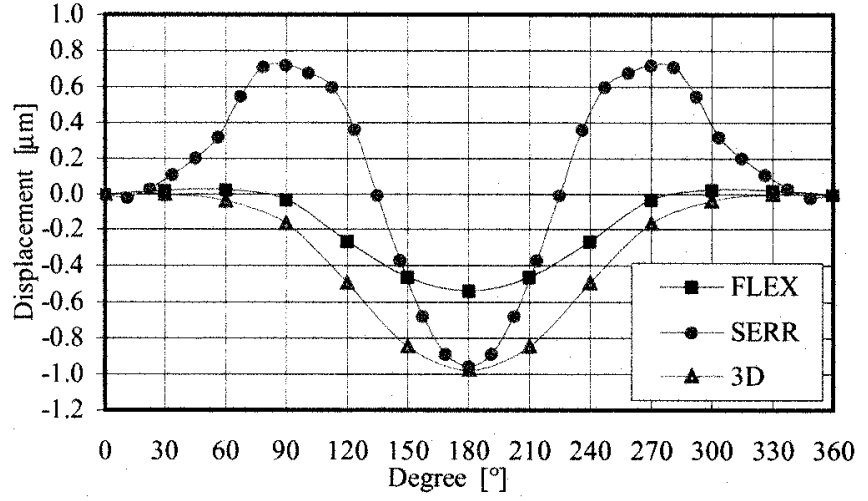

FIGURE 7

Pure bending, 50\% crack depth, $z$ displacement.

with respect to the pure bending situation (Figs. 12 and 13). It seems that the accuracy of the FLEX model is rather high, with few exceptions (for the axial displacement).

\section{Axial Loads}

Regarding the axial load, as shown in Figures 14, 15 and 16, good agreement was found for the horizontal and vertical displacements, which are due to coupling coefficients. The axial displacement was affected by an error of $6 \%$, which seems acceptable. The errors in torsional deflections were negligible; a comparison with the SERR results is not shown because they seemed unreasonable.

\section{RESULTS OF THE EXPERIMENT}

The results of a series of tests performed on a cracked specimen in various loading conditions are described. These tests were performed within the framework of common research activity with Electricite de France on a cracked rotor, and the cracked specimen was prepared by a research laboratory. The aim of the tests was to investigate the breathing behavior of the crack. In the vicinity of the crack's lips, strain gauges were

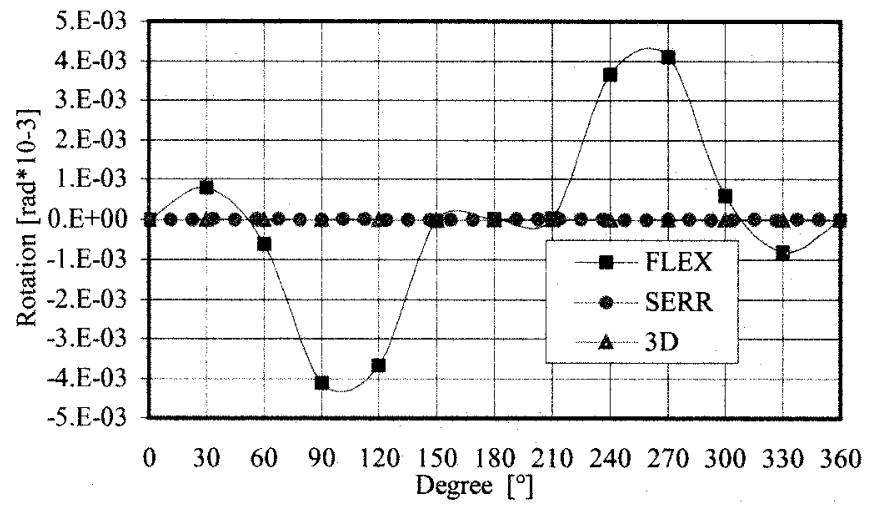

FIGURE 8

Pure bending, $50 \%$ crack depth, $\vartheta_{z}$ rotation.

Pure bending, 50\% crack depth, $\vartheta_{x}$ rotation. 


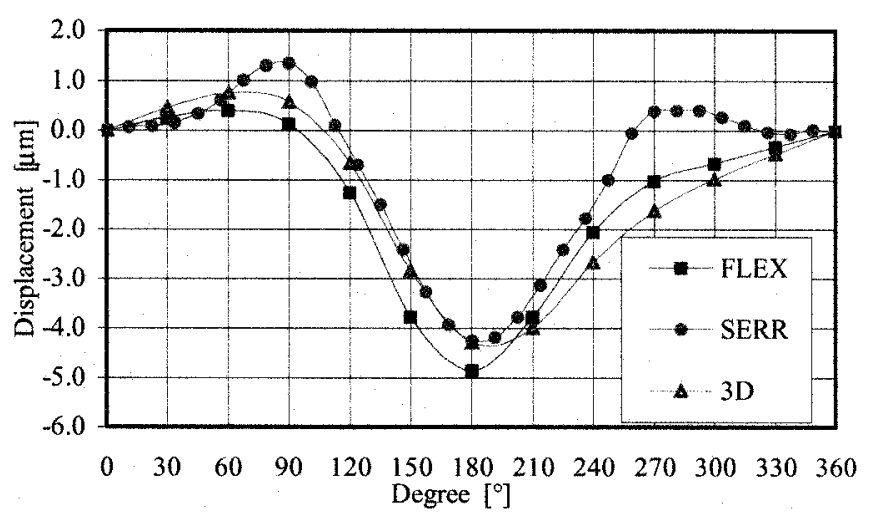

FIGURE 9

Bending and torsion, $50 \%$ crack depth, $\times$ displacement.

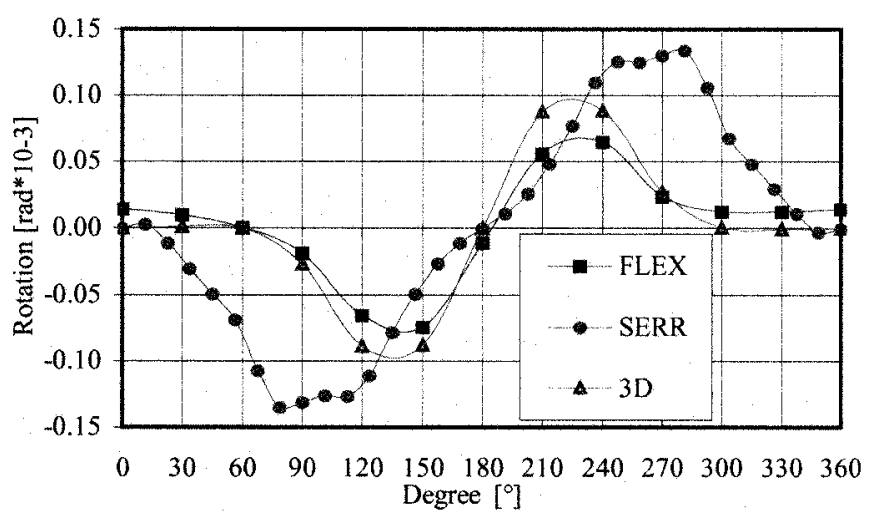

FIGURE 10

Bending and torsion, $50 \%$ crack depth, $\vartheta_{x}$ rotation.

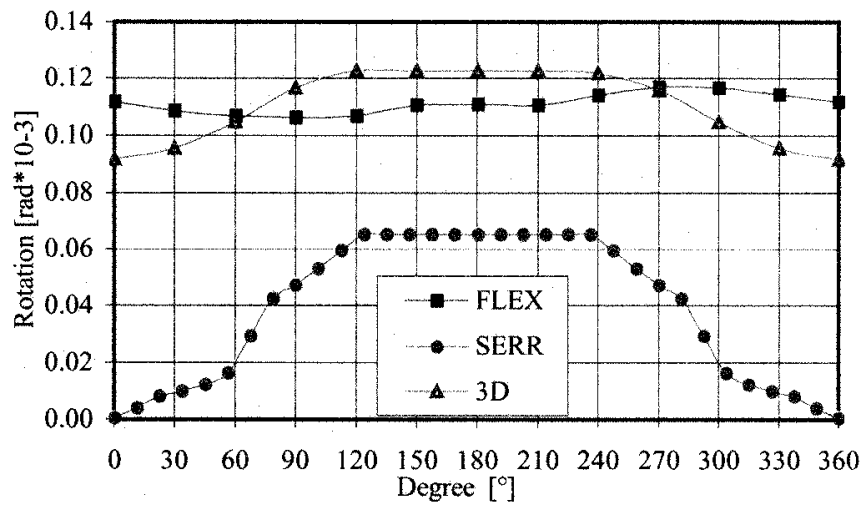

FIGURE 11

Bending and torsion, $50 \%$ crack depth, $\vartheta_{z}$ rotation.

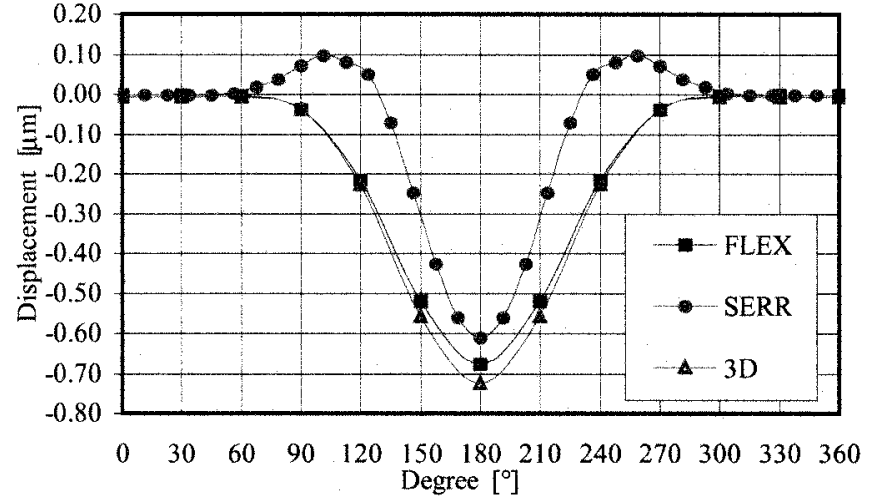

FIGURE 12

Bending and shear, $50 \%$ crack depth, $\times$ displacement.

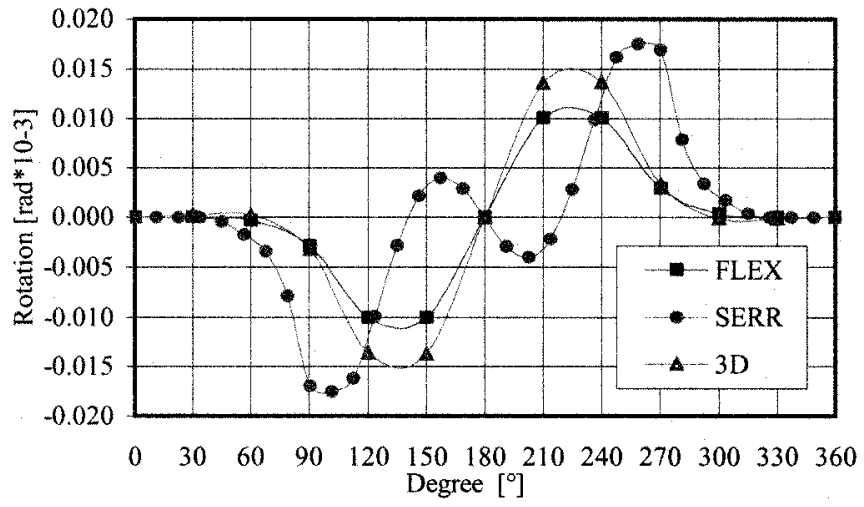

FIGURE 13

Bending and shear, 50\% crack depth, $\vartheta_{x}$ rotation.

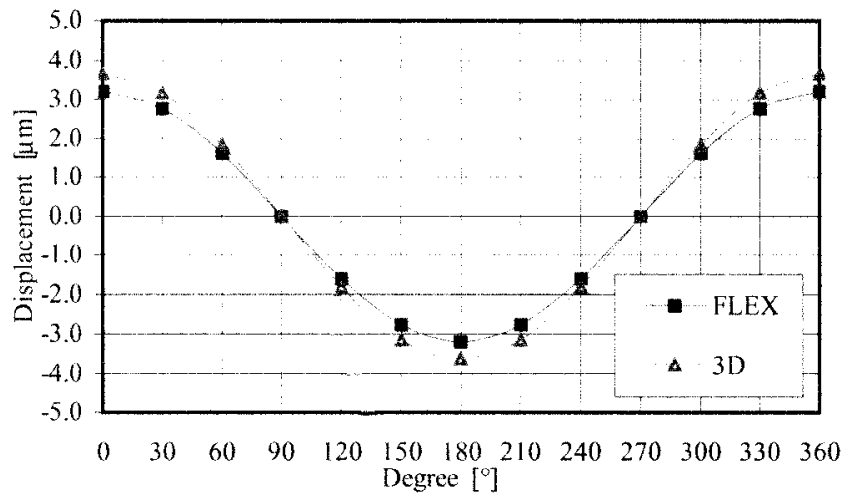

FIGURE 14

Axial load, 50\% crack depth, $\times$ displacement. 


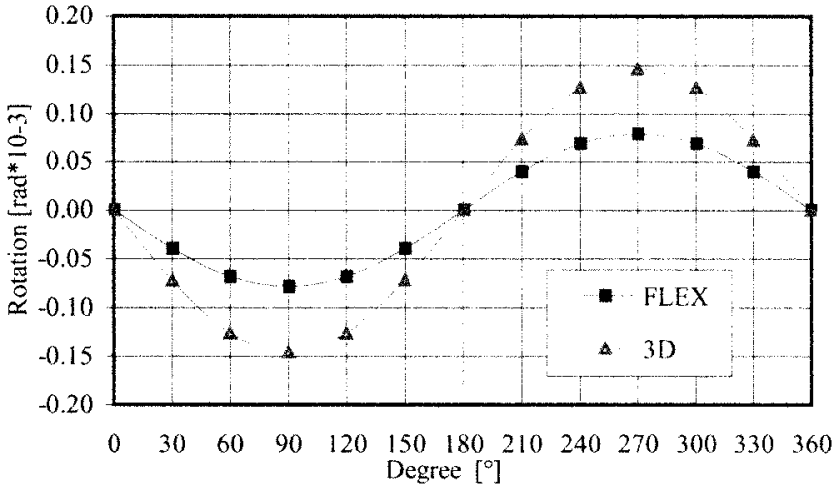

FIGURE 15

Axial load, 50\% crack depth, $\vartheta_{x}$ rotation.

applied in order to measure the stresses and to identify the load conditions (the value of the bending moment and the angular position of the load with respect to the crack) in which the lips start to lose contact. Then the measured stresses were compared to the stresses that had been calculated using the simplified 1D model.

\section{Description of the Test Rig}

The test specimen, schematically represented in Figure 17, was bolted to a shaft extension mounted on two roller bearings in a supporting structure. The shaft was then held by a pin in various angular positions.

At the opposite end of the specimen, several disks were bolted to the flange so various bending moments could be applied to the cracked specimen. In Figure 18, section A is the cracked section. The crack's profile, was obtained by means of ultra-sonic tests. Section B, located at a distance from the crack that is greater than the diameter of the specimen, was unaffected by the crack and served as the reference section. Sixteen different strain gauges were then applied in the positions shown in Figure 18.

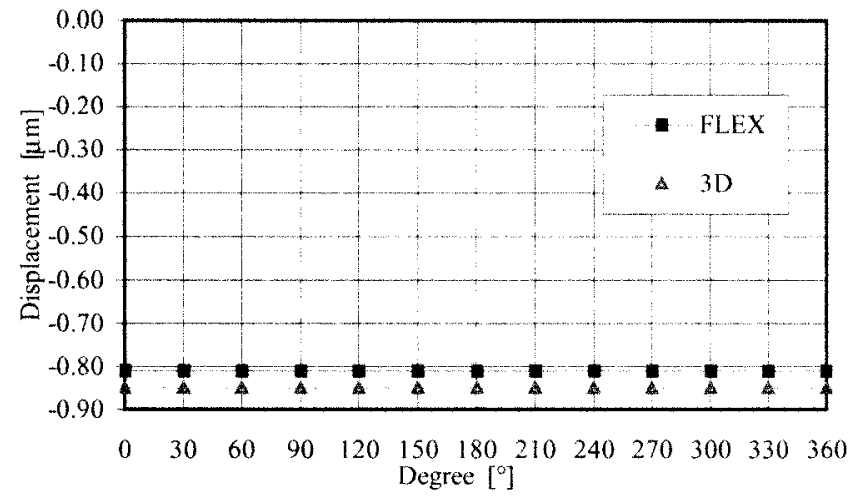

FIGURE 16

Axial load, 50\% crack depth, $z$ displacement.

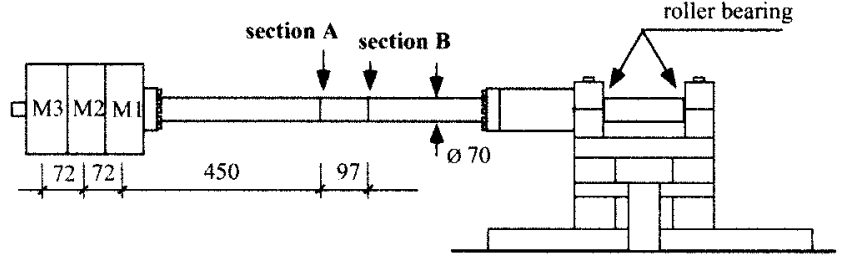

FIGURE 17

Schematic drawing of the test rig (dimensions are in $\mathrm{mm}$ ).

Strain gauge (SG)1 through SG11 were applied in correspondence with the crack's lips; SG16 was applied in the position opposite to the crack's lips in section A; and SG12 and SG15 were applied to section B as references.

\section{Measured Strains}

Measurement of strain was obtained from each strain gauge for the four different load conditions and in the various angular positions (every 15 degrees). Measurements are presented with respect to the direction of the vertical load.

Figures 19 through 23 show strain versus rotation angle, from 0 degree to 360 degrees, for the five strain gauges applied to the cracked specimen in the positions shown in Figure 18. The various loading conditions are specified in the diagrams.

The initial position ( 0 degree) corresponds to the crack's axis in the vertical position. With the lower loads (from $0 \mathrm{M}$ to $3 \mathrm{M}$ ) the pure sinusoidal shape of all the strain gauge measurements indicates that the crack is always closed. The positive strain on the crack's lips (elongation) is probably an "apparent" relative strain, not an absolute strain which, according to 3D calculations, cannot be positive. The strain gauges were applied to the specimen in conditions of external loads as close to zero as possible, but an internal bending moment probably developed during the propagation of the crack, and that would tend to hold the crack closed. The crack's lips are pressed against each other so that with no external loads, a compressive strain develops on

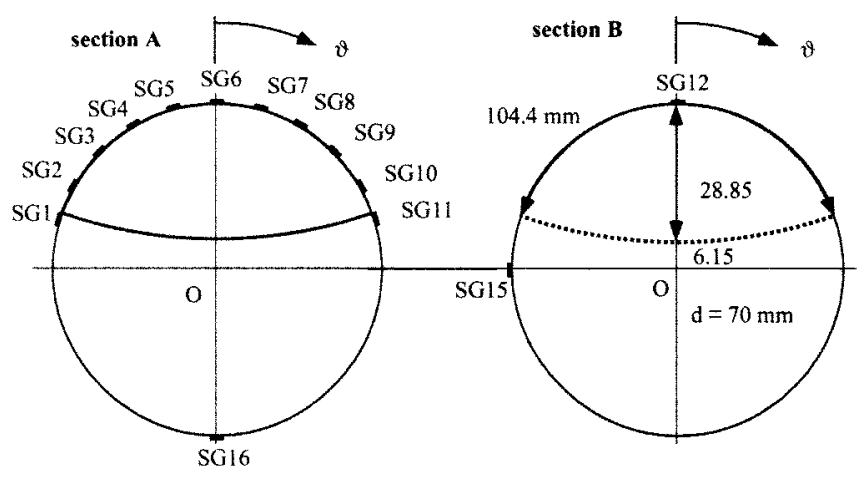

FIGURE 18

Strain gauge positions. 


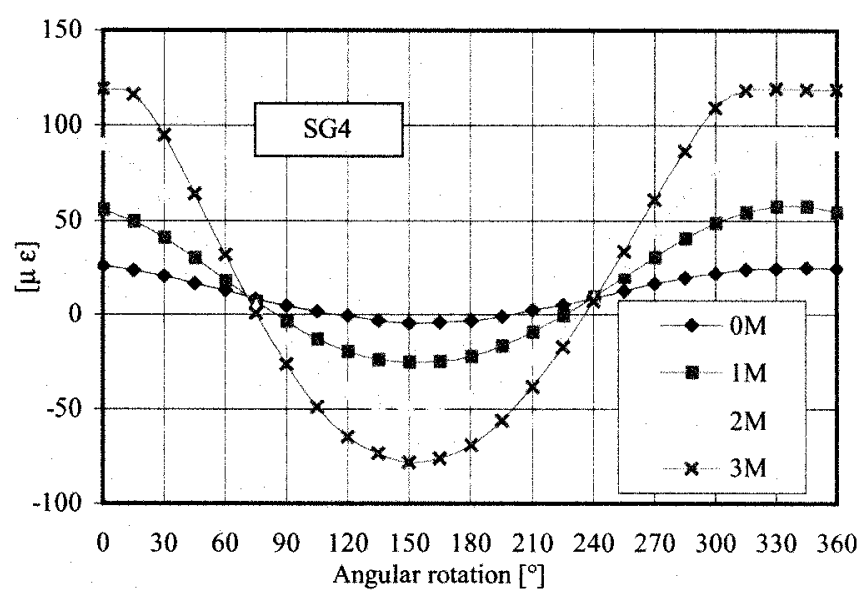

FIGURE 19

Strain gauge 4: strain versus rotation.

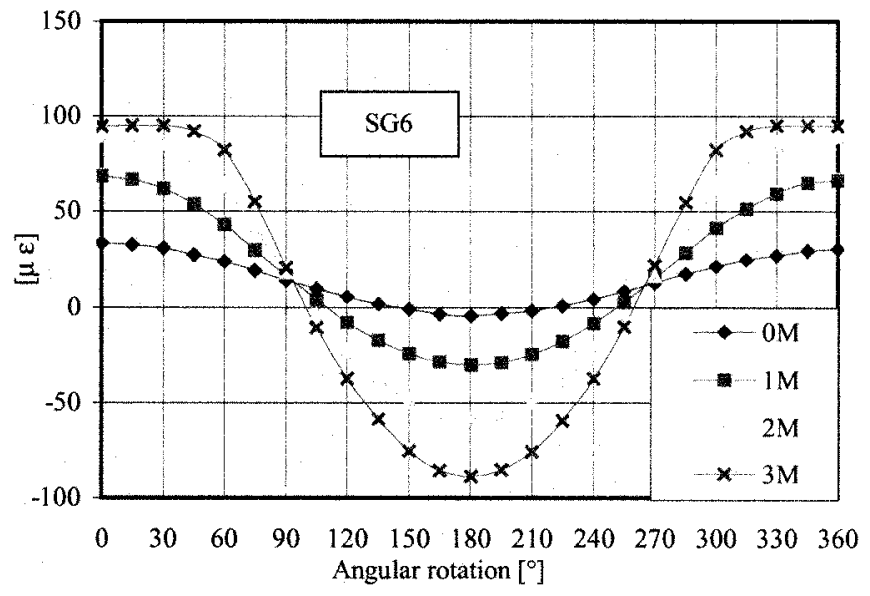

FIGURE 20

Strain gauge 6: strain versus rotation.

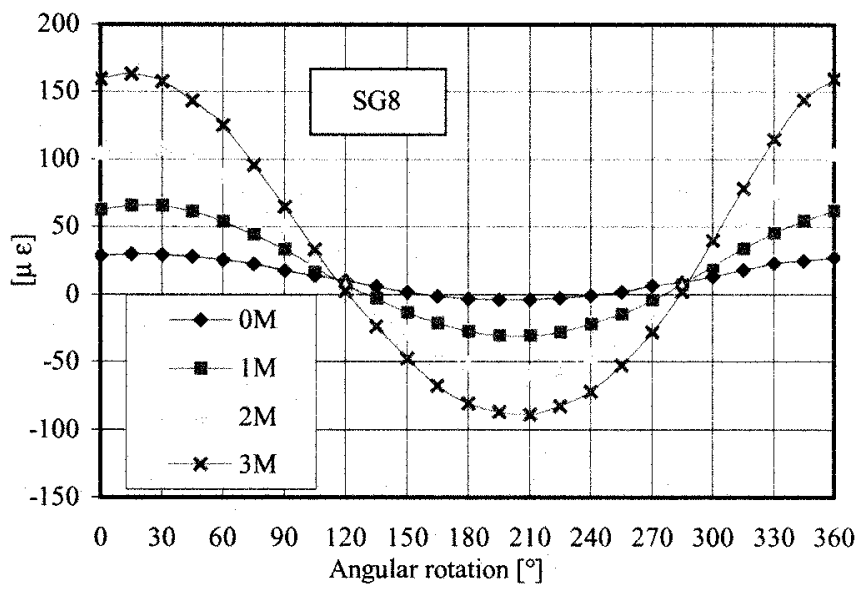

FIGURE 21

Strain gauge 8: strain versus rotation.

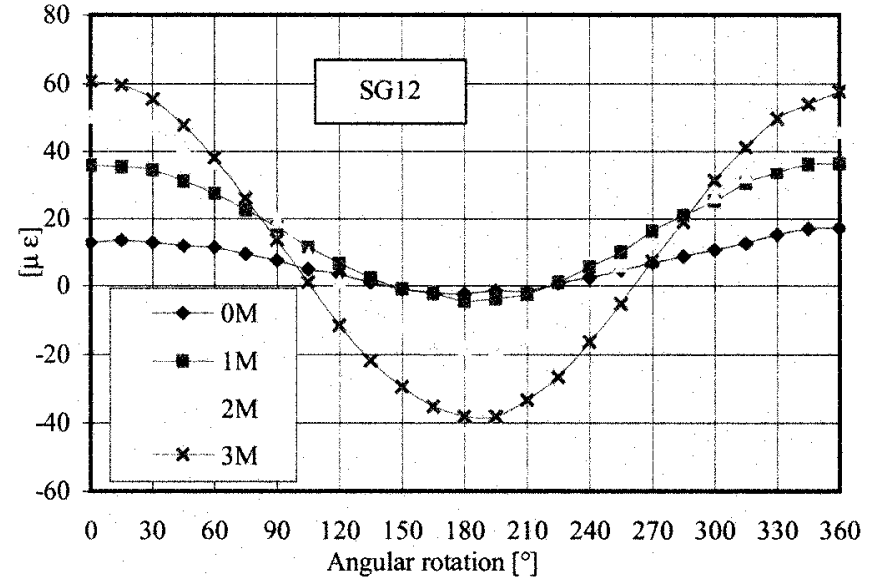

FIGURE 22

Strain gauge 12: strain versus rotation.

the lips, and that was not measured by the strain gauges. The crack's lips open only when the external bending moment overcomes the internal bending moment, and when the tensile stress of the external load equals the compressive stress of the internal bending moment. From this position on, the strain indicated by the gauge, which is the relative strain with respect to the initial reference situation, should remain constant with the increasing of the load; from this position on, the absolute strain should be zero.

This condition is fulfilled only with the highest load (3 M) and only for the gauges SG4, SG5, SG6, and SG7. At these measuring points the crack's lips open completely at several different angular postions. At the other measuring points on or close to the crack's lips, the strains always indicate a closed crack condition, probably because these points are close to the crack's tip or slightly outside the crack's lips.

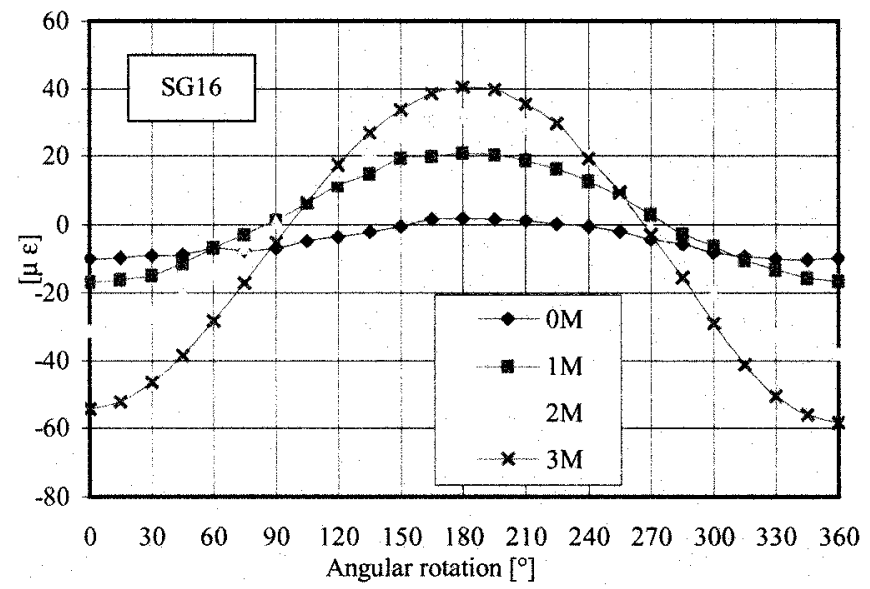

FIGURE 23

Strain gauge 16: strain versus rotation. 
TABLE 1

Loads, Bending Moments and Theoretical Strains

\begin{tabular}{|c|c|c|c|c|c|c|}
\hline \multirow[b]{2}{*}{ Loads } & \multicolumn{3}{|c|}{ Section A } & \multicolumn{3}{|c|}{ Section B } \\
\hline & $\begin{array}{c}\text { Bending } \\
\text { moments } \\
(\mathrm{Nm})\end{array}$ & $\begin{array}{c}\sigma \\
(\mathrm{MPa})\end{array}$ & $\begin{array}{c}\varepsilon \\
(\mu \varepsilon)\end{array}$ & $\begin{array}{c}\text { Bending } \\
\text { moments } \\
(\mathrm{Nm})\end{array}$ & $\begin{array}{c}\sigma \\
(\mathrm{MPa})\end{array}$ & $\begin{array}{c}\varepsilon \\
(\mu \varepsilon)\end{array}$ \\
\hline $0 \mathrm{M}$ & 37.4 & 1.1 & 5.4 & 53.0 & 1.6 & 7.6 \\
\hline $1 \mathrm{M}$ & 104.4 & 3.1 & 15.1 & 134.8 & 4.0 & 19.4 \\
\hline $2 \mathrm{M}$ & 181.9 & 5.4 & 26.2 & 226.4 & 6.7 & 32.6 \\
\hline $3 \mathrm{M}$ & 270.7 & 8.0 & 39.0 & 329.7 & 9.7 & 47.5 \\
\hline
\end{tabular}

Loads: 0 M, no additional mass; 1 M, 1 additional mass; 2 M, 2 additional masses; 3 M, 3 additional masses.

Another interesting point is made by these diagrams. The variation in theoretical strain with maximum load, as shown in Table 1 , is roughly between +40 and -40 microstrains, in correspondence with Section A, and the total peak-to-peak variation is 80 microstrains. In measuring point SG16 there is a minimum of 55 microstrains in the position where the crack is open (the stress distribution is apparently non linear) and there is a maximum of 40 microstrains in the position where the crack is closed (and the stress distribution is linear). This last value is exactly the theoretical value.

In SG11, a total variation of 88 microstrains indicates no nonlinear effects; the crack does not open and in this position no stress concentration occurs.

In SG1, a total variation of 130 microstrains indicates incoming nonlinear effects that increase by moving toward the crack's axis on both sides.

In SG10 and SG2, 150 peak-to-peak variations in microstrains are measured; in SG3, 180; and in SG8 and SG9, 250. In SG4 and SG5, the positive peak is flattened because the crack is opening; the minimum is -75 microstrains. Similar behavior is found at SG6 and SG7, where a minimum of -90 microstrains is reached.

The consistent increase in strain with respect to the theoretical values can be attributed to local stress concentration, which obviously depends on the varying depths of the lip's contact areas in correspondence with the various positions.

Finally, Figure 24 shows the calculated stress distribution for the 50\%-deep crack in the cases of an open and a closed crack. As can be seen, in the case of the closed crack and flat crack surfaces, a linear stress distribution is obtained with no stress concentration effects.

\section{Comparison with the Simplified 1D Model}

The 1D model was examined to ascertain its suitability for simulating the measured results of the experiment. The simplified model does not allow an accounting for an internal preload in the cracked area, nor for a contact occurring only on the lips of the crack (and not on the total cracked area). But an external

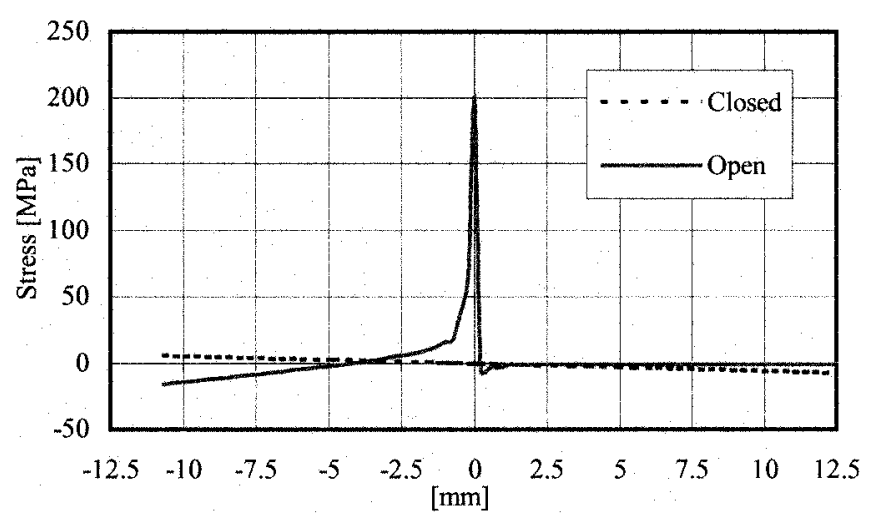

FIGURE 24

Calculated axial stress distribution for the 50\% crack depth in cases of an open and a closed crack.

bending moment that prevents the opening of the crack can be applied. This was done, and the value of the bending moment was tuned in order to have, when the maximum load was applied to the specimen, the angular position in which the crack starts to open in the position of SG6. The calculated theoretical stress does not take into account any stress concentration factor which, in this particular case, could be rather high because probably when the lips are closed, the contact occurs only in a small area below the outer surface of the lips.

With a stress concentration factor of 2.5 and a bending moment of $-230 \mathrm{Nm}$, the values shown in Figure 25 are obtained. They are compared with the values measured by SG6, which were shifted in order to have zero strain for an open crack.

The excellent agreement between measured and calculated results, although obtained after tuning the zero position and the stress concentration factor, confirms once again the validity of the proposed simplified 1D model.

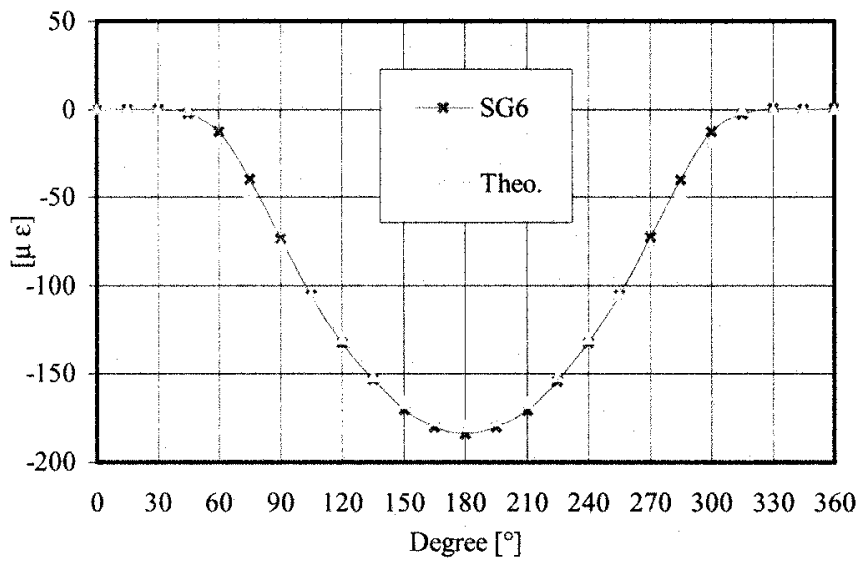

FIGURE 25

Theoretical strain values compared with measured strains in position SG6. 


\section{CONCLUSIONS}

Deflections of cracked beams according to six degrees of freedom due to different loading conditions were calculated using three different methods and were compared. The proposed linear 1D model shows good agreement with the nonlinear 3D model, both in the breathing behaviors (which influence the variations in stiffness of the beam) and in evaluating deflections. It is worth nothing that the analysis of each loading condition requires several hours of computation when using the 3D nonlinear model and only few seconds when using the 1D model. The breathing behavior has finally been investigated experimentally and some interesting effects deriving from crack propagation have been discovered.

\section{REFERENCES}

Bachschmid, N., Vania, A., and Audebert, S. 2000, March. A Comparison of different methods for transverse crack modeling in rotor systems. Proceeding of 8th ISROMAC-8, Publ. by Pacific Centre of Thermal-Fluids Engineering, Honolulu, Hawaii 96753, USA.

Dimarogonas, A. D. 1996. Vibration of cracked structures. A state-ofthe-art review. Engineering Fracture Mechanics 55:831-857.

Ferrari, G., and Campana, S. 1999. Comportamento Statico e Dinamico di un Rotore Criccato. (Master's thesis, Politecnico di Milano).

Gasch, R. 1993. A survey of the dynamic behavior of a simple rotating shaft with a transverse crack. Journal of Sound and Vibration 160:131-332.

Wauer, J. 1990. On the dynamics of cracked rotors: a literature survey. Applied Mechanics Reviews 43:13-17. 

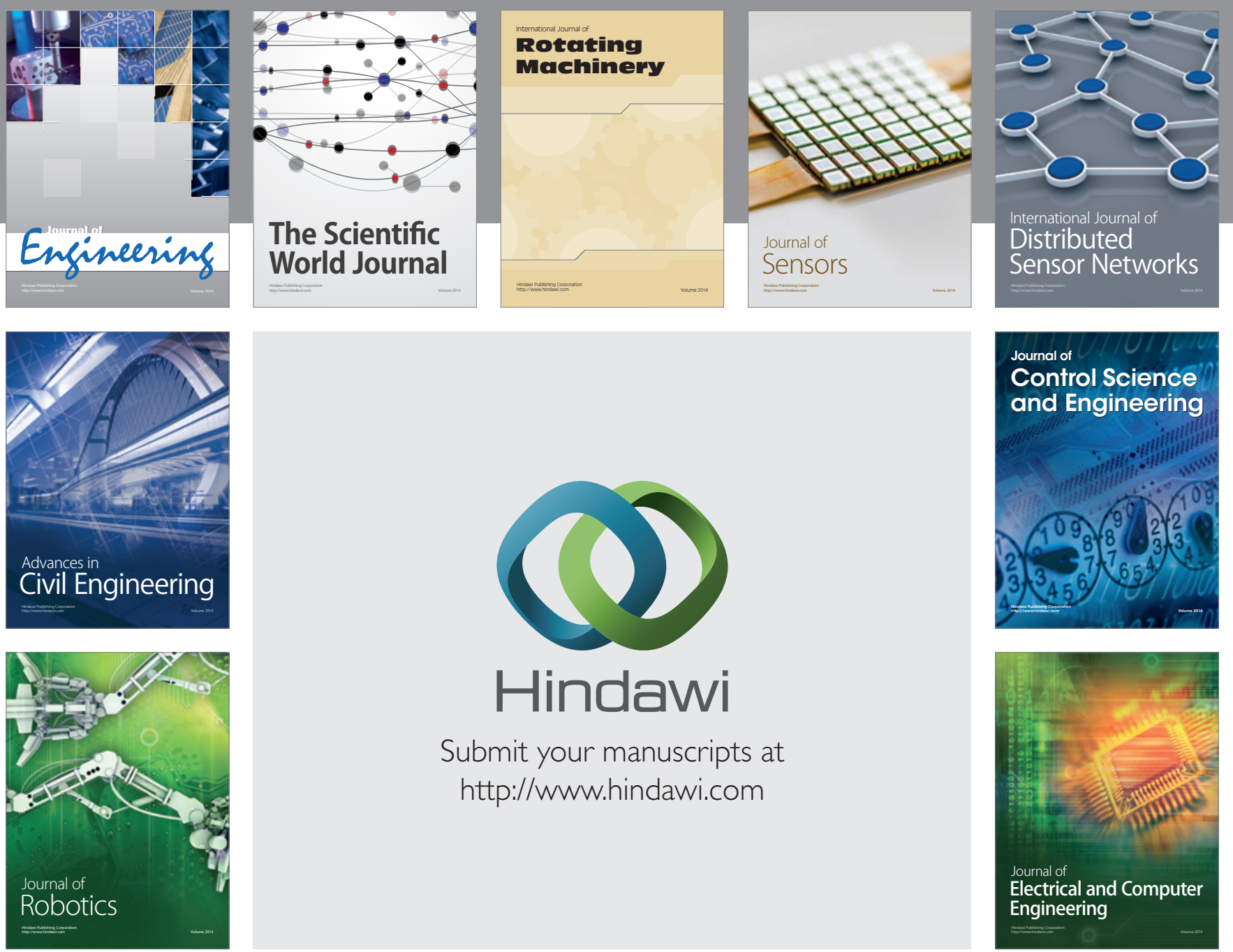

Submit your manuscripts at

http://www.hindawi.com
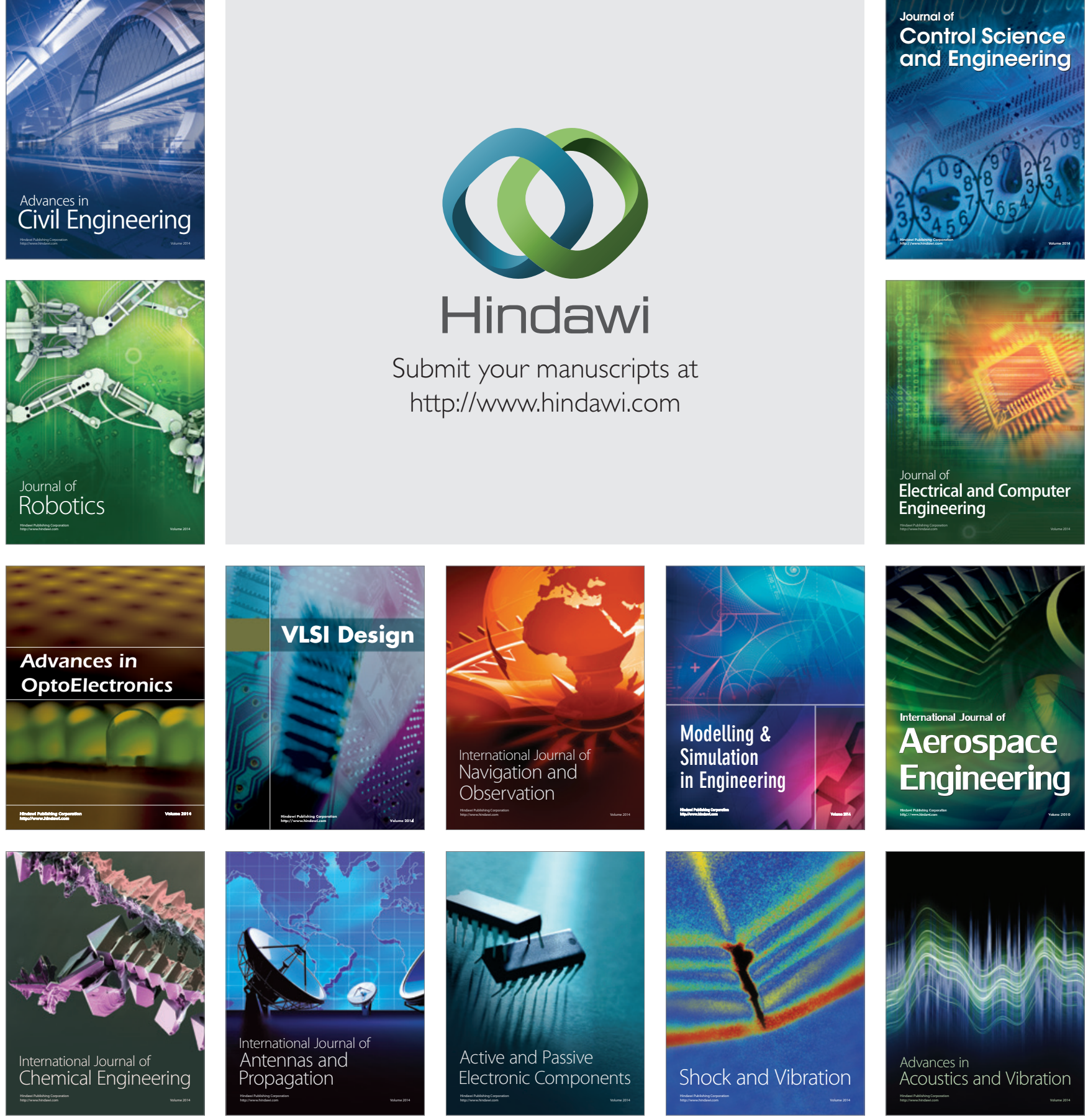